\title{
A pilot study evaluating the potential of Diffusional Kurtosis Imaging applied to Alzheimer's disease
}

\author{
Nicolas $R^{1,2,3}$, Saint-Aubert $L^{1,2}$, Gros-Dagnac $H^{1,2}$, Franceries $X^{1,2}$ and Pariente $J^{1,2}$ \\ ${ }^{1}$ INSERM, Imagerie cérébrale et handicaps neurologiques, UMR 825, F-31059 Toulouse, France \\ ${ }^{2}$ Université de Toulouse, UPS, Imagerie cérébrale et handicaps neurologiques, UMR 825, F-31059 Toulouse, France \\ ${ }^{3}$ INCIA, Aquitaine Institute for Cognitive and Integrative Neuroscience, UMR 5287, CNRS-Université Bordeaux, F-33400 Talence, France
}

\begin{abstract}
Aim: To estimate the potential of Diffusional Kurtosis Imaging (DKI), a method sensitive to microstructural damage for detecting the effects of Alzheimer's Disease (AD) compared to those of aging, in prodromal AD (PDAD).

Materials and methods: Young subjects $(N=4$, age $=25 \pm 3)$, old subjects $(N=7$, age $=65 \pm 8)$ and AD patients $(N=5$, age $=74 \pm 7)$ were recruited. Mean kurtosis $\left(K_{a p p}\right)$ in white matter (WM), grey matter (GM) were measured in the cerebellum, frontal lobe and temporal lobe, and differences were assessed with the non-parametric Kruskal-Wallis test.

Results: There was no age difference between PDAD patients and older subjects $(P>0.05)$. Mini-Mental State Examination (MMSE) scores of the patients were lower $(P<0.02)$ and comparatively in PDAD:

- Kurtosis $K_{a p p}$ was decreased in the temporal lobe WM only $(P<0.05)$.

There was a significant age difference in older subjects compared to young subjects, $(P<0.01)$ and comparatively in aging:

- $K_{a p p}$ was decreased in the frontal lobe WM only $(P<0.03)$.

Conclusion: $K_{a p p}$ decreases were found in the WM of the temporal lobe in PDAD and in the WM of the frontal lobe in aging, in accordance with previous studies. As PDAD microstructural and metabolic disturbances occur essentially in the temporal lobes where $K_{a p p}$ changes were also found, DKI might be a useful non-invasive method for the detection of Alzheimer's disease in the prodromal stage.
\end{abstract}

\section{Background and purpose}

The social and financial burden of Alzheimer's disease (AD) is a major health concern worldwide. When diagnosed with psychological evaluation, a large proportion of aged patients affected by mild cognitive impairment (MCI), and particularly those with amnesic MCI, can be suspected as having $\mathrm{AD}$ at the prodromal stage [1,2]. However, only pathological analysis on brain material, either using biopsy or post-mortem histological exam, can ascertain the diagnosis of $\mathrm{AD}$ in patients. $\mathrm{AD}$ is defined as the simultaneous detection in the brain of two microscopic tissue markers : amyloid plaque (AP, composed of fibrils of polymerized amyloid peptide $A \beta 40 / A \beta 42$ ) and neurofibrillary tangles (NFTs, composed of entangled hyperphosphorylated tau proteins). Glucose hypometabolism detected by 2-deoxy-2-(18F) fluoro-D-glucose PET (18F-FDG-PET) is a metabolic disturbance that is potentially associated with deleterious consequences of the presence of these abnormalities. In the $\mathrm{AD}$ brain, the anatomical course of glucose hypometabolism is similar to that of APs (or to that of NFTs). In AD, it is well-known that microscopic abnormalities and their consequences progress toward the temporal lobe and posterior cingulate cortex up to the temporal lobe and then attain the frontal cortex and the entire brain [3]. Predictors of PDAD are gradually declining memory, brain metabolic and functional disturbances and the presence in cerebrospinal fluid (CSF) of pathophysiological markers like free $\mathrm{A} \beta 42$ peptides and phosphorylated tau proteins [4].
Essentially, ${ }^{18} \mathrm{~F}$-FDG-PET and other PET methods have shown that in PDAD the hippocampus and the entorhinal cortex of the temporal lobe are the first part of the brain affected by the disease.

Diffusion magnetic resonance imaging (dMRI) is a non-invasive method able to help in the early $\mathrm{AD}$ diagnosis as it makes it possible to obtain markers providing information at the meso- and microstructural levels. Changes in dMRI parameters in $\mathrm{AD}$ have been detected in the hippocampus and temporo-parietal areas, i.e. in the same locations as those where the microstructural abnormalities of the disease occur [5-7]. However, although current dMRI parameters have been shown empirically to be good AD hallmarks. Indeed, it has also long been shown that indexes like the Apparent Diffusion Coefficient (ADC), Mean Diffusivity (MD) and Fractional Anisotropy (FA) are sensitive to the effects of aging in tissue, their changes being essentially detected in the frontal lobe [5-8]. To improve the sensitivity of dMRI to microstructural damage, advanced dMRI acquisition uses images acquired with very high diffusion-weighting factors ( $b$-values).

Correspondence to: Renaud Nicolas, INSERM, Imagerie cérébrale et handicaps neurologiques, UMR 825, F-31059 Toulouse, France, Tel: 05569192 36; E-mail: renaud.nicolas@u-bordeaux.fr

Received: August 22, 2017; Accepted: September 20, 2017; Published: September 25, 2017 
Recently, a method closely related to q-space imaging, Diffusional Kurtosis Imaging (DKI). DKI performs the truncated expansion in power of the b-value of the diffusion-weighting-dependent MRI signal attenuation [11]. DKI parameters remain representative of information conveyed by water displacement at the tenths of a micrometer scale [11]. It was recently demonstrated that DKI can be used to probe microstructural damage in MCI- and AD-affected brain [12,13]. Mean (angularly-averaged) apparent kurtosis $K_{a p p}$ has been shown to be one of the most sensitive DKI markers for early AD diagnosis [12,13]. Mean kurtosis $\left(K_{a p p}\right)$ has been found to be promisingly sensitive as an early $\mathrm{AD}$ marker as it detects $\mathrm{AD}$-related changes in the temporal lobe but not in the frontal lobe [12].

The main goal of the present study was to propose to discriminate $\mathrm{AD}$-related changes (or their consequences) and the effects of aging with a fast DKI method estimating only angularly-averaged kurtosis and diffusion coefficients.

\section{Materials and methods}

\section{Subjects \& patients}

The ethics committee of South-West France and Overseas French departments, France, (Comité de Protection des Personnes Sud-Ouest et Outre Mer I-II) approved the present study protocol. Written informed consent was obtained from every subject/patient before participation. The population comprised young subjects $(N=4$, age $=25 \pm 3$ years $)$, older subjects $(N=7$, age $=65 \pm 8$ years, MMSE $=28 \pm 1.1)$ and PDAD patients $(N=5$, age $=74 \pm 7$ years, $\mathrm{MMSE}=25.8 \pm 1.4)$. PDAD patients were recruited according to Dubois's AD diagnostic criteria $[1,2,4]$. This diagnostic criteria included episodic memory deficiency assessed by a full psychological evaluation, MMSE $(\leq 27)$, atrophy of the internal temporal structures detected on $T_{1}$-weighted images, presence of free $A \beta_{42}$, or abnormal ratio of free phosphorylated/ unphosphorylated tau proteins in CSF [2,4], and temporo-parietal hypometabolism as detected by ${ }^{18} \mathrm{~F}-\mathrm{FDG}$ PET. WM hyperintensities detected on $T_{2}$-weighted images and generalized brain atrophy in $T_{1}$ weighted images were exclusion criteria.

\section{Brain imaging}

${ }^{18} \mathrm{~F}$-FDG-PET images of the subject in the case study were acquired at $3.5 \mathrm{MBq} / \mathrm{kg}$ of ${ }^{18} \mathrm{~F}-\mathrm{FDG}$ with a Biograph 6 True Point Hirez (Siemens, Knoxville, USA). dMRI was performed with a $3 \mathrm{~T}$ magnet (Philips, Best, The Netherlands) equipped with an 8-channel head coil and with a magnetic gradient field system able to reach $40 \mathrm{mT} / \mathrm{m}$. Diffusionweighted (DW) images were acquired with increasing $b$-factors $\left(b=0,200,500,1000,1800,2500 \mathrm{~s} / \mathrm{mm}^{2}\right)$. The diffusion gradient edge separation/diffusion gradient duration ratio used was $\Delta / \delta=34.5 / 25 \mathrm{~ms}$, with $\mathrm{TE} / \mathrm{TR}=70 / 4212 \mathrm{~ms}$. Sensitivity Encoding for Fast MRI (SENSE) was used as a parallel imaging method with a SENSE factor of 2. Then, 31 slices at $2 \times 2 \times 4 \mathrm{~mm}^{3}$ spatial resolution were acquired without any gap, covering the entire brain volume. The MRI scanner provided the angular average of DW images (geometric average of images acquired with diffusion magnetic field gradients oriented in read, phase and slice directions). Duration of the dMRI protocol was 6 minutes, a duration compatible with its use in multi-modal hospital clinical studies of $\mathrm{AD}$.

\section{DW Image registration}

The diffusion-weighted-dependent attenuation of the MRI signal was designated as $S(b)$, whereas the signal without diffusion weighting was designated as $S_{0}$. The $S(b)$ images were submitted to a brain extraction using Otsu's histogram-based segmentation [14].
Then, the subject's motion and eddy current effects on $S(b)$ were corrected respectively by a global/rigid and a local/elastic registration implemented in Matlab with $S_{0}$ as a reference image. Registration then provided $S(b)$ images realigned on $S_{0}$ images [15].

\section{Calculation of diffusion maps}

Both the choice of the number of $b$-values used and the number of spatial directions of diffusion measures used have an impact on the duration of the DKI protocol. Cognitively impaired patients cannot be maintained for long in the magnet. Owing to time constraints in common clinical routines, our DKI acquisition protocol was simplified in comparison to the work presented in references [12,13]. Mean kurtosis was previously demonstrated to be the most pertinent index for $\mathrm{AD}$ detection compared to other tensorial DKI metrics [12,13]. The angular-averaged diffusion-weighted-dependent attenuation of the MRI signal $(S(b))$ signal obtained in each voxel was fitted with the DKI model [11]:

$$
S(b)=S_{0} \cdot \exp \left[-b \cdot D_{a p p}+1 / 6\left(b^{2} \cdot D_{a p p}^{2} \cdot K_{a p p}\right)\right]
$$

where $b$ is the diffusion-weighting factor ( $b$-value). The mean diffusion coefficient $D_{a p p}$ represents the contribution of the Gaussian diffusion of the signal. In a first approximation, $D_{a p p}$ could be considered as analogous to $M D$ or $A D C$. Mean kurtosis $K_{a p p}^{a p p}$ is representative of non-Gaussian diffusion contribution [11]. The fitting convergence of the experimental signal and of equation (1) was implemented with a simple least-square regression method using the Nelder-Mead simplex algorithm (fminsearch $\mathrm{Matlab}^{\star}$ function). Computer-assisted calculation for the whole brain (image registration and computation of DKI parameters) took $\sim 10 \mathrm{~min}$ with a computer equipped with a $2 \mathrm{x} 4$ core processor $\left(\mathrm{Intel}^{\circledR} \mathrm{Xeon}^{\oplus} \mathrm{CPU}\right.$ L5520 functioning at $2.27 \mathrm{GHz}$ ).

\section{Volumes of interests (VOI) \& localized values}

Masks of brain substructures: Echo planar images were submitted to the FSL segmentation algorithm FAST (FMRIB Automated Segmentation Tool), leading to anatomically separated binary masks attributed to cerebrospinal fluid (CSF), grey matter (GM) and white matter (WM) [16,17]. Relative anatomical volumes (segmented volumes of CSF, GM and WM relative to the subject imaged full brain volume) were calculated. The potential changes in these relative volumes with aging and with PDAD (both causing macroscopic atrophy) were also investigated.

Anatomical mask of brain lobes: In $K_{a p p}$ parametric images, twelve contiguous slices were selected to present the same anatomical features for young subjects, old subjects and PDAD patients. The anatomical locations of these slices were defined by the shapes of the ventricle, by deep GM localization, and by the global brain anatomy of the gyri compared with a brain atlas. In these $K_{a p p}$ parametric images which provide a very good anatomical contrast, a second class of mask defining anatomic brain lobe regions were obtained by a single operator trained in neuroanatomy who manually defined large anatomic VOIs. The groups' status was blinded when performing the VOI analysis. Segmentation separated the brain into large anatomic areas corresponding to the anatomical locations of the cerebellum, frontal lobe and temporal lobe, in a similar manner to that previously reported [18]. VOIs were non-ambiguously delimited according to basic anatomy (cerebellum) and to the central sulci (frontal lobe) and the lateral sulci (temporal lobe). Temporal lobe VOIs included the enthorhinal cortex and hippocampus. The cerebellum is a reference structure that is classically not expected to be affected by PDAD at this early stage. 
Parcellation \& obtainment of localized values: The two types of binary masks obtained were combined by multiplication. The resulting combined masks were multiplied pixel-by pixel by $K_{a p p}$ and $D_{a p p}$ maps. This finally led to quantitative average localized values designed by $<D_{a p p}>$ and $<K_{a p p}>$ for GM and WM in the cerebellum, temporal and frontal lobes.

\section{Statistical analysis}

All variables: MMSE, ages, relative brain volumes of segmented structures, spatially-averaged values $\left(\left\langle D_{a p p}\right\rangle\right.$ and $\left.\left\langle K_{a p p}\right\rangle\right)$ were compared pairwise (group by group) by a non-parametric KruskalWallis test implemented natively in the R statistical software [19,20]. The Kruskal-Wallis test is an extension of the Wilcoxon test and makes it possible to test differences between small samples with potentially unequal numbers of observations and/or unequal variance [19]. Separately for each anatomical area, $\left\langle D_{a p p}\right\rangle$ and $\left\langle K_{a p p}>\right.$ values were treated pairwise between old $v s$ young subjects and between PDAD patients $v s$ old subjects to assess their potential changes. Statistical significance was considered at the conventional level $P<0.05$.

\section{$K_{a p p}$ templates}

To obtain $K_{a p p}$ maps in a common brain space, we performed a global and local elastic registration [15] of $K_{a p p}$ maps. $K_{a p p}$ maps were then subject-averaged, providing preliminary $K_{a p p}$ anatomic templates for young, aged and PDAD subjects expressed in a common reference frame (Figure 1).

\section{Results}

\section{Age comparisons}

No significant age differences were found between PDAD patients $v s$ older subjects. However, mini-Mental State Examination (MMSE) scores were significantly lower in PDAD patients than in old subjects $(P<0.02)$, In aged subjects, MSME $\geq 27$ attested to the absence of significant cognitive deterioration in older subjects. Young subjects were significantly younger than older subjects and PDAD patients $(P<0.01)$.

\section{Study of groups}

Estimation of effects of atrophy: The CSF, GM and WM segmented volumes relative to each subject's total imaged brain volume are shown in Table $1 \mathrm{~A}$ and the statistical significance of the volume differences between patients and subjects are displayed in Table 1B. Indirect effects of macroscopic atrophy caused by age and/or AD were marked essentially by an increase in the pixels segmented as CSF (Table 1B). A specific decrease in the global volume of the WM relative to the imaged brain volume was found in PDAD patients' brains compared to aged subjects' ones $(P<0.05$, Table $1 \mathrm{~B})$.

Analysis of DKI maps: Subject-averaged images of $K_{a p p}$ templates are shown in Figure 1. A dramatic increase in ventricular volume attested to a strong effect of atrophy in PDAD brains. There was a less marked and more diffuse intensity of $K_{a p p}$ in the WM of PDAD patients. $K_{a p p}$ maps in WM have a more uniform appearance in the WM of PDAD patient than in that of aged subjects, owing to the absence of high $K_{a p p}$ values in some zones of the WM (Figure 1).

No differences in $D_{a p p}$ or $K_{a p p}$ due to PDAD or aging were found in the cerebellum. In PDAD patients $v s$ older subjects, $K_{a p p}$ was significantly decreased in the temporal lobe WM $(P<0.05)$. Considering the effect of aging, a significant $K_{a p p}$ decrease was observed in the frontal lobe WM of older subjects $(P<0.03$, Table 1$)$.
Table 1. Estimation of the effects of atrophy. A) Percentage of segmented volumes relative to each individual imaged brain volume (mean \pm standard deviation). B) Statistical significance of the difference in relative volume between groups by paired Kruskal-Wallis test. The non-significant (NS) values are indicated by "-".

\begin{tabular}{|c|c|c|c|}
\hline $\begin{array}{c}\text { A) mask volume } \\
\text { relative to brain } \\
\text { volume }\end{array}$ & CSF & GM & WM \\
\hline Young subject & $9 \pm 3 \%$ & $44 \pm 5 \%$ & $47 \pm 9 \%$ \\
\hline Older subjects & $24 \pm 2 \%$ & $34 \pm 2 \%$ & $42 \pm 5 \%$ \\
\hline PDAD patients & $33 \pm 4 \%$ & $32 \pm 6 \%$ & $35 \pm 2.5 \%$ \\
\hline B) Probability $\boldsymbol{P}$ & & & \\
\hline Older $v$ Young & $P<0.01$ & - & - \\
\hline PDAD $v$ s Older & $P<0.05$ & - & $P<0.05$ \\
\hline
\end{tabular}
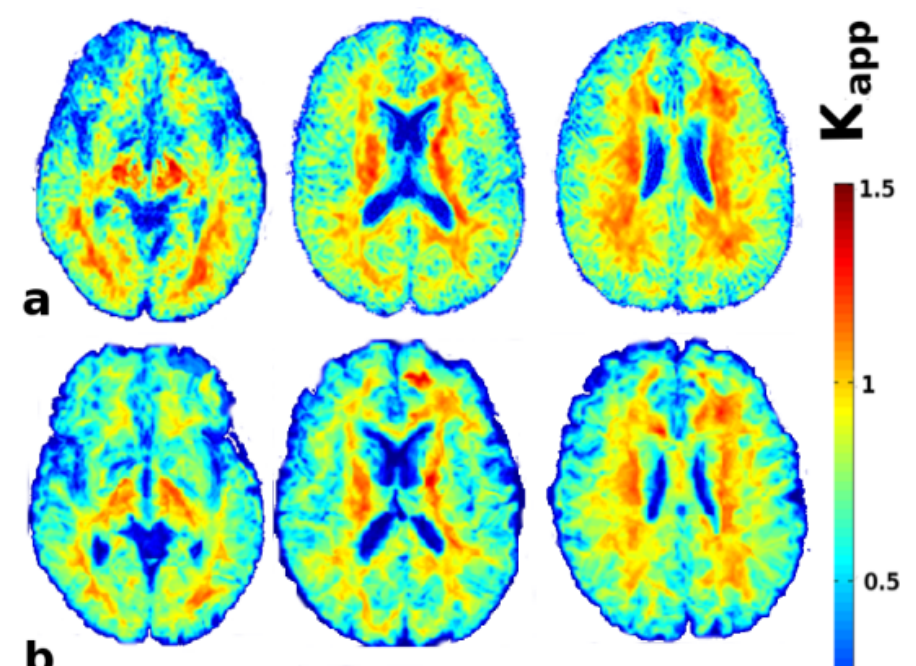

1

b
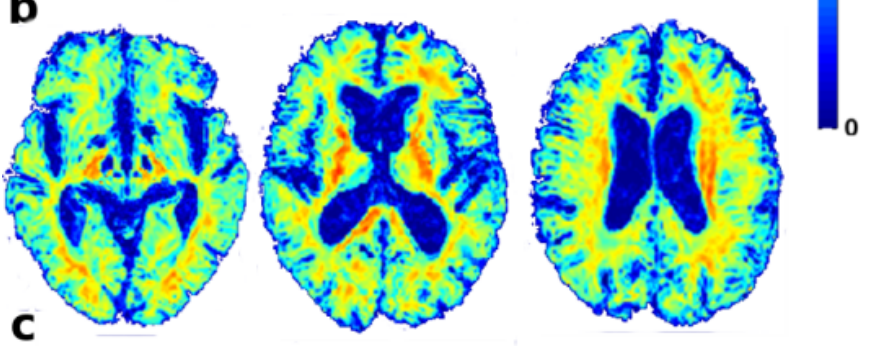

Figure 1. Templates of subject-averaged $K_{a p p}$ maps (three non-adjacent slices). a) Young subject-averaged $K_{a p p}$ maps b) Old subject-averaged $K_{a p p}$ maps c) PDAD patients averaged $K_{a p p s}$ maps. $K_{a p p}$ is unitless. Zero $K_{\text {app }}$ values indicate Gaussian diffusion, non-zero $K_{a p p}$ values indicate the intensity of the non-Gaussian diffusion (i.e., sensitized to microstructural features).

\section{Case study}

The case study patient showed a strongly asymmetric left glucose hypometabolism in the hippocampus (Figure 2a, arrowhead), in the entorhinal cortex and in the temporal lobe (Figure 2a, arrow) as detected by ${ }^{18} \mathrm{~F}$-FDG-PET. This glucose hypometabolism in the entire left temporal lobe was associated with an asymmetrically lateralized $K_{a p p}$ decrease in the hippocampus and entorhinal cortex of the left temporal lobe that was visible to the naked eye (Figure $2 b$ ). When comparing histograms of $K_{a p p}$ maps for the segmented right and left temporal lobes, the main peak of the histogram (representing WM with elevated $K_{a p p}$ ) in the affected left temporal lobe (peak maximum at 54 pixels) was lower than in the right temporal lobe (peak maximum at 80 pixels) (Figure $2 b^{\prime}$ '). Unlike $K_{a p p}$ (Figure $2 b$ ), the other diffusion indexes $A D C$ or $D_{a p p}$ (Figure $2 c$ ) did not show any evident asymmetric changes in their images (Figure 2c) or in their histogram (data not shown). 

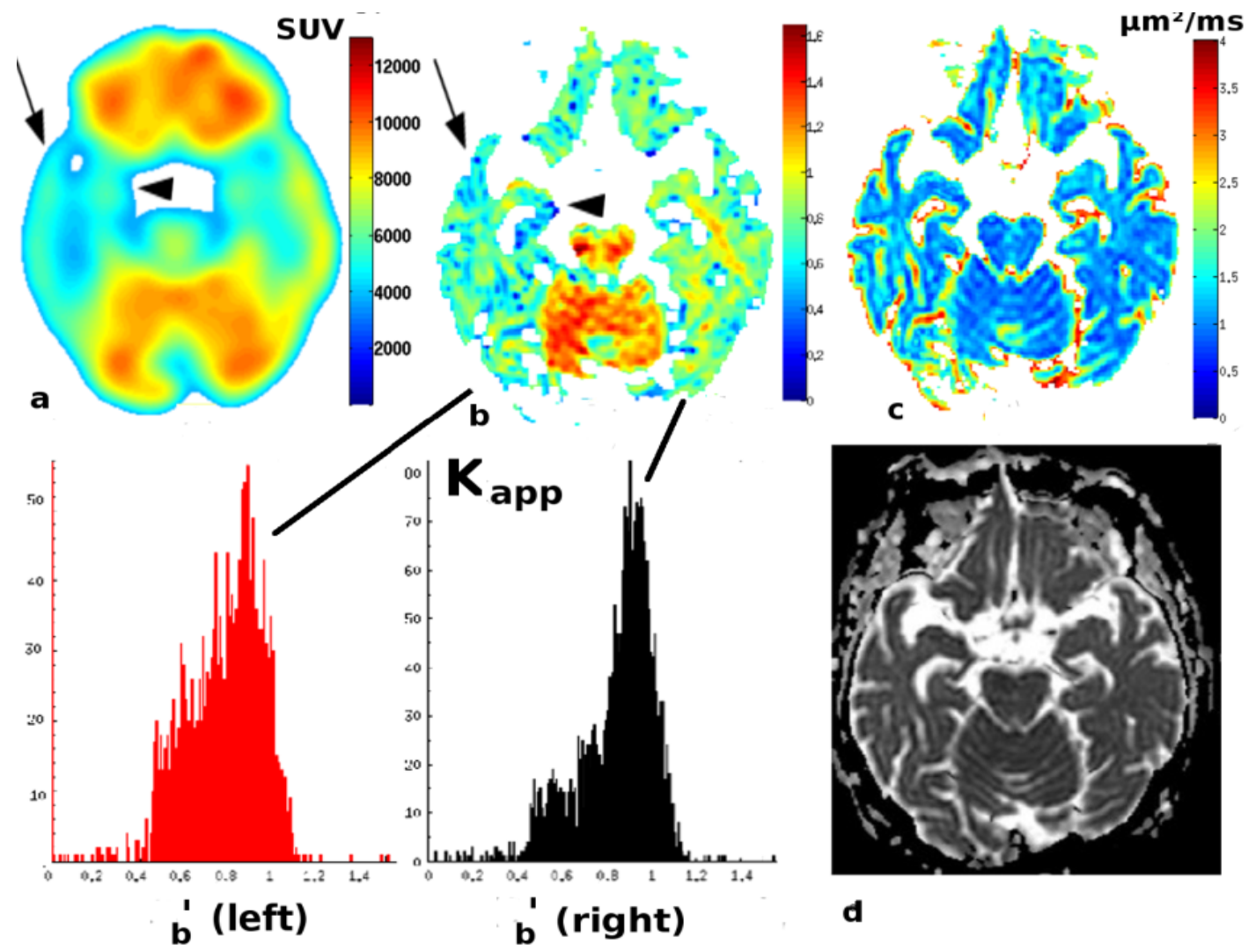

Figure 2. Images of the PDAD patient case study:

a) ${ }^{18}$ F-FDG PET image (units: SUV, Standardized ${ }^{18}$ F-FDG Uptake Values) showing a lateral hypometabolism in left temporal lobe (arrow) and in left hippocampus (arrowhead). b) Apparent kurtosis $K_{a p p}$ map showing kurtosis decrease in left temporal lobe (arrow) and in left hippocampus (arrowhead). In b': Histograms of kurtosis $K_{a p p}$ images for affected left temporal lobe and hippocampus (b', red), more preserved right temporal lobe and hippocampus.

c) $D_{a p p}$ maps $\left(\mu \mathrm{m}^{2} / \mathrm{ms}\right)$ obtained from DKI model;

d) $A D C$ measures calculated by the MRI scanner.

\section{Discussion}

The following section discusses the results by separating the effects of PDAD and aging. For the diffusion parameter $K_{a p p}$, the main finding of the study was that a statistically significant $K_{a p p}$ decrease was found only in the temporal lobe WM of PDAD patients ( $v s$ age-matched controls) in a region typically affected by the disease, the temporal lobe. This reproduces recent findings demonstrating that $K_{a p p}$ is an efficient marker of AD-related microstructural alterations in such anatomical areas [12,13]. A significant decrease in $K_{a p p}$ was also observed in manually segmented temporal WM and the anterior corona radiata in $\mathrm{AD} v s$ age-matched controls, whereas no changes were found in $K_{a p p}$ in segmented frontal WM [12]. DTI studies exploring WM structure have confirmed that in $\mathrm{AD}$, abnormalities in FA and MD occur mostly in the posterior regions such as the parahippocampal gyrus, the anterior temporal WM, the splenium of the corpus callosum and the posterior cingulum $[5-7,21,22]$.

On the other hand, the effect of aging was evidenced by a statistically significant $K_{\text {app }}$ decrease in the frontal WM of aged subjects. An agedependent $K_{a p p}^{a p p}$ decrease has also been observed during normal human brain aging in the frontal area, similar to findings reported in this work [23]. DTI studies have confirmed that in normal aging, FA and MD abnormalities occur in the frontal lobes $[8,24]$, especially in the frontal $\mathrm{WM}$, in the anterior cingulum and in the genu of the corpus callosum [21-22,24]. Therefore, our findings (concerning $K_{a p p}$ ) that the effects of PDAD are visible in temporal lobe and that the effects of aging are visible in the frontal lobe are in accordance with previous studies. Our findings are very similar to those concerning the observed changes in $K_{a p p}$ in WM of AD patients [12,13].

In $\mathrm{AD}$ studies, it is necessary to include as a validation the estimation of the effect of macroscopic atrophy. A specific effect of tissue atrophy in PDAD patients was detected as a significant decrease in the area of WM relative to the total imaged brain volume $(P<0.05$, Table 1$)$. This strongly suggests that atrophy specifically caused by PDAD occurs in the WM, or affects WM density, as also suggested by Figure 1, and also by histograms in Figure 2. The indirect effects of atrophy caused by $\mathrm{PDAD}$ and by age were marked essentially by an increase in the volume segmented as CSF $(P<0.05, P<0.01$, respectively, Table 1$)$.

A second internal validation of our results concerns the use of an anatomical area as a reference. In the cerebellum, no $K_{a p p}$ or $D_{a p p}$ changes specific to PDAD or aging were detected. As motor control is not specifically affected by aging or by PDAD, this reinforced our hypothesis that the effects detected in the frontal and temporal lobes are specific to aging and disease-related changes and are not false positives.

\section{Conclusion}

This pilot study shows that DKI has potential interest for studying $\mathrm{AD}$ at the prodromal stage. Microstructural changes of an unknown nature but specific to PDAD were detected by $K_{a p p}$ in the temporal lobe, a site where microstructural changes specific to PDAD are found. The specificity of the $K_{a p p}$ parameter was related to its location in the 
temporal lobe, not to the change in the parameter itself, because the effects of aging were found to be a similar $K_{\text {opp }}$ decrease but located in the frontal lobe of older subjects. $K_{a p p}$ might thus be an index to discriminate the effects of PDAD and aging on the basis of location (temporal lobe $v s$ frontal lobe). The results also suggest that the $K_{a p p}$ decrease in PDAD might be more tightly linked to microscopic changes than the increases in $D_{a p p}$.

The case study results suggest that there might be a spatial correlation between $K_{a p p}$ and ${ }^{18} \mathrm{~F}$-FDG-PET for assessing metabolic disturbances potentially associated with microstructural damage in PDAD. In particular, $K_{a p p}$ is inherently sensitive to microstructural abnormalities like axonal properties and it may be spatially associated with more conventional metabolic AD markers. Decreases in $K_{a p p}$ might reflect specific microstructural abnormalities in PDAD that are potentially associated with glucose metabolic disturbances. One hypothesis is that $K_{a p p}$ decreases reflect a loss of the structural complexity of WM. This could occur either by the lesser presence in $\mathrm{AD}$ brain of the crossing of neuronal fibers or by partial axonal degeneration. These two hypotheses can lead to the reported changes in diffusion properties ( $K_{a p p}$ decreases). This axonal degeneration that occurs in PDAD is due to the destructuring of the microtubular architecture of axons which results from the hyperphosphorylation of tau proteins, and might be linked to the $K_{\text {app }}$ decrease observed in WM of $\mathrm{AD}$ patients. The exact determinism that makes DKI a microstructurally sensitive method of detection of the brain lesions in $\mathrm{AD}$ patients now needs to be examined in a larger sample of patients and with the help of multi-modal imaging methods.

\section{References}

1. Dubois B, Albert ML (2004) Amnestic MCI or prodromal Alzheimer's disease? Lancet Neurol 3: 246-248. [Crossref]

2. Dubois B, Feldman HH, Jacova C, Dekosky ST, Barberger-Gateau P, et al. (2007) Research criteria for the diagnosis of Alzheimer's disease: revising the NINCDSADRDA criteria. Lancet Neurol 6: 734-746. [Crossref]

3. Mosconi L, Berti V, Glodzik L, Pupi A, De Santi S, et al. (2010) Pre-clinical detection of Alzheimer's disease using FDG-PET, with or without amyloid imaging. J Alzheimers Dis 20: 843-854. [Crossref]

4. Herukka SK, Hallikainen M, Soininen H, Pirttilä T (2005) CSF Abeta42 and tau or phosphorylated tau and prediction of progressive mild cognitive impairment. Neurology 64: 1294-1297. [Crossref]

5. Kantarci K, Jack CR, Xu YC et al (2001) Mild cognitive impairment and Alzheimer disease: regional diffusivity of water. Radiology 219:101-107.

6. Ito S (2008) Brain Diffusion Changes in Patients Diagnosed with Alzheimer's Disease. Curr Med Imag Rev 4: 226-230.

7. Chua TC, Wen W, Slavin MJ, Sachdev PS (2008) Diffusion tensor imaging in mild cognitive impairment and Alzheimer's disease: a review. Curr Opin Neurol 21: 83-92. [Crossref]
8. Nusbaum AO, Tang CY, Buchsbaum MS, Wei TC, Atlas SW (2001) Regional and global changes in cerebral diffusion with normal aging. AJNR Am J Neuroradiol 22: 136-142. [Crossref]

9. Yoshiura T, Mihara T, Tanaka A (2003) High $b$ value diffusion-weighted imaging is more sensitive to white matter degeneration in Alzheimer's disease. NeuroImage 20: 413-419.

10. Mayzel-Oreg O, Assaf Y, Gigi A (2007) High $b$-value diffusion imaging of dementia application to vascular dementia and Alzheimer disease. J Neurol Sci 257:105-113.

11. Jensen JH, Helpern JA, Ramani A, Lu H, Kaczynski K (2005) Diffusional kurtosis imaging: The quantification of non-gaussian water diffusion by means of magnetic resonance imaging. Magn Reson Med 53: 1432-1440. [Crossref]

12. Falangola MF, Jensen JH, Tabesh A (2013) Non-Gaussian diffusion MRI assessment of brain microstructure in mild cognitive impairment and Alzheimer's disease. Magn Reson Imaging 31: 840-846.

13. Gong NJ, Wong CS, Chan CC, Leung LM, Chu YC (2013) Correlations between microstructural alterations and severity of cognitive deficiency in Alzheimer's disease and mild cognitive impairment: a diffusional kurtosis imaging study. Magn Reson Imaging 31: 688-694. [Crossref]

14. Otsu N (1979) A threshold selection method from gray-level histograms. IEEE Trans Sys Man Cyber 9: 62-66.

15. Periaswamy S, Farid H (2003) Elastic registration in the presence of intensity variations. IEEE Trans Med Imaging 22: 865-874. [Crossref]

16. Hadjiprocopis A, Rashid W, Tofts PS (2005) Unbiased segmentation of diffusionweighted magnetic resonance images of the brain using iterative clustering. Magn Reson Imaging 23: 877-885.

17. Yoshiura T, Wu O, Zaher A, Reese TG, Sorensen AG (2001) Highly Diffusionsensitized MRI of brain: dissociation of Gray and White Matter. Magn Reson Med 45: 734-740.

18. Yamada K, Sakai K, Akazawa K, Sugimoto N, Nakagawa M, et al. (2013) Detection of early neuronal damage in CADASIL patients by q-space MR imaging. Neuroradiology 55: 283-290.

19. Vargha A, Delaney HD (1998) The Kruskal-Wallis Test and Stochastic Homogeneity. J Educ Behav Stat 23:170-192.

20. Dean CB, Nielsen JD (2007) Generalized linear mixed models: a review and some extensions. Lifetime Data Anal 13: 497-512. [Crossref]

21. Stahl R, Dietrich O, Teipel SJ, Hampel H, Reiser MF, et al. (2007) White matter damage in Alzheimer disease and mild cognitive impairment: assessment with diffusion-tensor MR imaging and parallel imaging techniques. Radiology 243: 483-492. [Crossref]

22. Stebbins GT, Murphy CM (2009) Diffusion tensor imaging in Alzheimer's disease and mild cognitive impairment. Behav Neurol 21: 39-49. [Crossref]

23. Falangola MF, Jensen JH, Babb JS, Hu C, Castellanos FX, et al. (2008) Age-related non-Gaussian diffusion patterns in the prefrontal brain. $J$ Magn Reson Imaging 28: 1345-1350. [Crossref]

24. Salat DH, Lee SY, Yu P, Setty B, Rosas HD, et al. (2009) DTI in development and aging, Chapter 10, Diffusion MRI: from quantitative measurement to in vivo neuroanatomy. Johansen-Berg H, Behrens TE, Elsevier, ISBN: 978-0-12-374709-9.

Copyright: (C2017 Nicolas R. This is an open-access article distributed under the terms of the Creative Commons Attribution License, which permits unrestricted use, distribution, and reproduction in any medium, provided the original author and source are credited. 